\title{
STRICT COMPLEX CONVEXITY
}

\author{
ROBERT CARMIGNANI
}

\begin{abstract}
The strictly subharmonic and strictly plurisubharmonic functions are characterized using distribution theory, namely, the distributional Laplacian and the distributional complex Hessian. Then the analogous results for strictly convex functions are given.
\end{abstract}

Introduction. In this work we first characterize strict subharmonicity using the Laplace operator in the distributional sense (Theorem 1.6). From this and the well-known fact that plurisubharmonicity is characterized by the distributional complex Hessian form we obtain necessary and sufficient conditions (Theorems 1.7 and 1.10) for a function to be strictly plurisubharmonic. Example 1.9 shows however that the two conditions are not equivalent.

Finally, we give the analogous results for strictly convex functions.

0 . Notation. For $\Omega$ an open set in $\mathbf{C}^{n}$ (which we identify with $\mathbf{R}^{2 n}$ ) $\mathscr{D}(\Omega)$ is the space of all infinitely differentiable functions with compact support in $\Omega$, whose dual space is the set of distributions in $\Omega, \mathcal{D}^{\prime}(\Omega)$. For a distribution $T \in \mathscr{D}^{\prime}(\Omega), T \geqslant 0$ means that $T(\phi) \geqslant 0$ for all nonnegative $\phi \in \mathscr{D}(\Omega)$ (i.e. $T$ is positive $\left.{ }^{1}\right)$ and $T>0$ means that $T(\phi)>0$ for all nonnegative $\phi(\phi$ $\not \equiv 0)$ in $\mathscr{Q}(\Omega)$ (i.e. $T$ is strictly positive ${ }^{2}$ ).

$L^{1}(\Omega)$ denotes the space of Lebesgue integrable functions on $\Omega$ and a.e. means almost everywhere with respect to Lebesgue measure.

Arbitrary elements of $\mathbf{C}^{n}$ and $\mathbf{R}^{n}$ are denoted respectively by $z=\left(z_{1}, \ldots\right.$, $\left.z_{n}\right)$ and $x=\left(x_{1}, \ldots, x_{n}\right) \cdot|z|=(z \bar{z})^{1 / 2}$ where $\bar{z}$ is the complex conjugate of $z$.

Int $A$ and $\bar{A}$ denote respectively the interior and the closure of $A$.

\section{Complex convexity.}

Definition 1.1. A function $u$ defined in an open set $\Omega \subset \mathbf{C}$ and with values in $[-\infty,+\infty)$ is called strictly subharmonic if

(a) $u$ is upper semicontinuous in $\Omega$.

(b) For every compact set $K \subset \Omega$ and every continuous function $h$ on $K$

Received by the editors September 3, 1974.

AMS (MOS) subject classifications (1970). Primary 32F05, 31A05, 26A51, 46F99; Secondary $10 \mathrm{C} 99$.

Key words and phrases. Strictly plurisubharmonic functions, strictly subharmonic functions, strictly convex functions, complex Hessian forms, real Hessian forms, differential quadratic forms, positive distributions, distributional complex Hessian, distributional real Hessian.

${ }^{1} T$ is positive semi-definite.

${ }^{2} T$ is positive definite. 
which is harmonic in Int $K$ and is $\geqslant u$ on the boundary of $K$ we have $u<h$ in Int $K$.

Definition 1.2. A function $u$ defined in an open set $\Omega \subset \mathbf{C}^{n}$ is said to be strictly plurisubharmonic in $\Omega$ if

(a) $u$ is upper semicontinuous in $\Omega$.

(b) For arbitrary $z \in \Omega$ and $w \in \mathbf{C}^{n}$, the function $\tau \rightarrow u(z+\tau w)$ is strictly subharmonic in $\{\tau \in \mathbf{C}: z+\tau w \in \Omega\}$.

For $\phi \in \mathscr{D}(\Omega)$ the distributional complex Hessian $H(u ; \phi)$ of a locally integrable function $u$ in an open set $\Omega \subset \mathbf{C}^{n}$, determines a Hermitian form

$$
(H(u ; \phi) a, \bar{a})=\sum_{j, k=1}^{n} \int_{\mathbf{C}^{n}} u(z) \frac{\partial^{2} \phi(z)}{\partial z_{j} \partial \bar{z}_{k}} a_{j} \bar{a}_{k} d \lambda(z), \quad a \in \mathbf{C}^{n},
$$

and $\lambda$ denotes the Lebesgue measure. Now for each nonnegative $\phi \in \mathscr{D}(\Omega)(\phi$ $\not \equiv 0),(1.3)$ is positive semidefinite (or respectively positive definite) if and only if for each fixed nonzero $a \in \mathbf{C}^{n}$, the distribution defined by $(H(u ; \phi) a, \bar{a})$ as $\phi$ ranges over $\mathscr{D}(\Omega)$ is positive (or respectively strictly positive).

We shall use the following well-known result (for a proof see [5, p. 80]).

THEOREM 1.4. Let $u \not \equiv-\infty$ be plurisubharmonic in an open set $\Omega \subset \mathbf{C}^{n}$. Then (1.3) is positive semidefinite for each nonnegative $\phi \in \mathscr{D}(\Omega)$. Conversely, if $u$ is locally integrable and (1.3) is positive semidefinite for each nonnegative $\phi$ $\in \mathcal{D}(\Omega)$, then there exists a unique plurisubharmonic function $\nu$ in $\Omega$ such that $u=\nu$ a.e. in $\Omega$.

Let $\Delta$ be the Laplace operator in $\mathbf{R}^{2}$. For any locally integrable function $u$ in an open set $\Omega \subset \mathbf{C}$, let $\Delta u$ be the distribution defined by $\Delta u(\phi)=\int u \Delta \phi, \phi$ $\in \mathscr{D}(\Omega)$. Then Theorem 1.4 when $n=1$ is equivalent to

THEOREM 1.5. If $u \not \equiv-\infty$ is subharmonic in $\Omega$, then $\Delta u \geqslant 0$ in $\Omega$. Conversely, if $u$ is locally integrable in $\Omega$ and $\Delta u \geqslant 0$ in $\Omega$, then there exists a unique subharmonic function $\nu$ in $\Omega$ such that $u=\nu$ a.e. in $\Omega$.

A proof of this theorem is given in [5, p. 67] and in [2, p. 19].

THEOREM 1.6. If $u \not \equiv-\infty$ is strictly subharmonic in an open set $\Omega \subset \mathbf{C}$, then $\Delta u>0$ in $\Omega$. Conversely, if $u$ is locally integrable in $\Omega$ and $\Delta u>0$ in $\Omega$, then there exists a strictly subharmonic function $\nu$ in $\Omega$ such that $u=\nu$ a.e. in $\Omega$.

Proof. Assume that $u \not \equiv-\infty$ is strictly subharmonic in $\Omega$. Then by Theorem 1.5 we have that $\Delta u \geqslant 0$. Since a positive distribution is defined by a unique positive measure, there exists a unique positive measure $\mu$ such that $\Delta u(\phi)=\int \phi d \mu$, for all $\phi \in \mathcal{D}(\Omega)$. Now suppose that $\Delta u$ were not strictly positive, that is, suppose there exists a nonnegative $\Psi \in \mathcal{D}(\Omega)(\Psi \not \equiv 0)$ such that $\Delta u(\Psi)=0$. Then $\{z: \Psi(z)>0\}$ is a nonempty set of $\mu$-measure zero. Let $\Omega_{1}$ be any domain in $\{z: \Psi(z)>0\}$. Then $u(\phi)=\int \phi d \mu=0$ for all $\phi$ $\in \mathscr{D}\left(\Omega_{1}\right)$. This means that $u$ is a distributional solution of $\Delta u=0$ in $\Omega_{1}$. By Weyl's lemma there exists a harmonic function $w$ such that $u=w$ a.e. in $\Omega_{1}$, 
and since $u$ is subharmonic $u \equiv w$ in $\Omega_{1}$. But this contradicts the strict subharmonicity of $u$.

On the other hand, assume that $u$ is locally integrable in $\Omega$ and that $\Delta u>0$ in $\Omega$. Then by Theorem 1.5, $u$ equals a subharmonic function $\nu$ a.e. in $\Omega$. Now suppose that $\nu$ is not strictly subharmonic in $\Omega$. Then there exists an open set $\Omega_{2} \subset \Omega$ such that $\nu$ is harmonic in $\Omega_{2}{ }^{3}$ This implies that $\Delta \nu(\phi)=\Delta u(\phi)=0$ for all $\phi \in \mathscr{D}\left(\Omega_{2}\right)$, which contradicts the strict positivity of $\Delta u$. This completes the proof.

REMarK. Theorem 1.6 is valid for strictly subharmonic functions in $\mathbf{R}^{n}$, where $\mathbf{C}$ is replaced by $\mathbf{R}^{n}$ and $\Delta$ is the Laplacian in $\mathbf{R}^{n}$.

THEOREM 1.7. Let $u \neq \equiv-\infty$ be strictly plurisubharmonic in an open set $\Omega \subset \mathbf{C}^{n}$. Then (1.3) is positive definite for each nonnegative $\phi(\phi \not \equiv 0)$ in $\mathscr{D}(\Omega)$.

Proof. By Theorem 1.4, (1.3) is positive semidefinite for each nonnegative $\phi \in \mathscr{D}(\Omega)$. Now suppose that there exists a nonnegative $\Psi(\Psi \not \equiv 0)$ in $\mathscr{D}(\Omega)$ such that (1.3) is not positive definite, that is, suppose that $\left(H(u ; \Psi) a_{0}, \bar{a}_{0}\right)=0$ for some nonzero $a_{0} \in \mathbf{C}^{n}$. Let $T$ be the distribution defined by $\left(H(u ; \phi) a_{0}\right.$, $\left.\bar{a}_{0}\right)$ as $\phi$ ranges over $\mathscr{D}(\Omega)$. Then $T$ is positive but not strictly positive since $T(\Psi)=0$. The positivity of $T$ implies that there exists a positive measure $\mu$ such that $T(\phi)=\int \phi d \mu$. Let $\Omega_{1}$ be any domain in $\{z: \Psi(z)>0\}$. Then $T(\phi)=0$ for all $\phi \in \mathscr{D}\left(\Omega_{1}\right)$. Let $L_{a}$ be the differential operator defined by

$$
L_{a}=\sum_{j, k=1}^{n} a_{j} \bar{a}_{k} \frac{\partial^{2}}{\partial z_{j} \partial \bar{z}_{k}} .
$$

Then $u$, thought of as a distribution, satisfies $L_{a_{0}}(u)=T$. Hence $u$ is a distributional solution in $\Omega_{1}$ of the homogeneous equation $L_{a_{0}}=0$. This means that the regularizations $u_{\varepsilon}$ of $u$ are classical solutions of this equation in $\Omega_{1}$ for $\varepsilon$ sufficiently small. It is well known (see [2, p. 45]) that for any compact set $K \subset \Omega, u_{\varepsilon}$ is plurisubharmonic on a neighborhood of $K$ for all $\varepsilon$ smaller than some $\varepsilon_{0}$. Furthermore, the $u_{\varepsilon}$ decrease and converge to $u$. Since $\bar{\Omega}_{1} \subset \Omega$, an application of the chain rule when $\varepsilon \leqslant \varepsilon_{0}$ yields

$$
\begin{aligned}
L_{a}\left(u_{\varepsilon}(z)\right) & =\sum_{j, k=1}^{n} \frac{\partial^{2} u_{\varepsilon}(z)}{\partial z_{j} \partial \bar{z}_{k}} a_{j} \bar{a}_{k} \\
& =\frac{\partial^{2} u_{\varepsilon}\left(z^{0}+\tau a\right)}{\partial \tau \partial \bar{\tau}},
\end{aligned}
$$

for all $z^{0}+\tau a=z \in \Omega_{1}$ where $\tau \in \mathbf{C}$. Since $L_{a_{0}}(u)=0$ in $\Omega_{1}$ we see from (1.8) that for each $\varepsilon \leqslant \varepsilon_{0}$ the function $\tau \rightarrow u_{\varepsilon}\left(z^{0}+\tau a_{0}\right)$ is harmonic in some open set in $\mathbf{C}$ for each $z^{0} \in \Omega_{1}$. For fixed $z^{0} \in \Omega_{1}$ and $\varepsilon \leqslant \varepsilon_{0}$, each $u_{\varepsilon}\left(z^{0}\right.$ $\left.+\tau a_{0}\right)$ is harmonic in a fixed open set $\Omega_{0} \subset \mathbf{C}$. Now $u_{\varepsilon}\left(z^{0}+\tau a_{0}\right)$ converges to $u\left(z^{0}+\tau a_{0}\right)$ in $L^{1}\left(K_{0}\right)$ as $\varepsilon \rightarrow 0$, for any compact set $K_{0} \subset \Omega_{0}$ (see [3, p. 3] for a proof). So by Hölder's inequality we obtain that

${ }^{3}$ This follows from the maximum principle. 


$$
\begin{aligned}
u_{\varepsilon}\left(z^{0}+\tau a_{0}\right) \frac{\partial^{2} \phi(\tau)}{\partial \tau \partial \bar{\tau}} \rightarrow u\left(z^{0}+\tau a_{0}\right) & \frac{\partial^{2} \phi(\tau)}{\partial \tau \partial \bar{\tau}} \\
& \text { in } L^{1}\left(\Omega_{0}\right) \text { for each } \phi \in \mathscr{D}\left(\Omega_{0}\right) .
\end{aligned}
$$

Thus by Weyl's lemma (or also by Theorems 1.5 and 1.6) we can conclude that $u\left(z^{0}+\tau a_{0}\right)$ is harmonic in $\Omega_{0}$, which contradicts the strict plurisubharmonicity of $u$. This proves the theorem.

If $u$ is a locally integrable function in $\Omega$ such that (1.3) is positive definite for each nonnegative $\phi \in \mathcal{D}(\Omega)(\phi \neq \equiv 0)$, then by Theorem 1.4 there exists a plurisubharmonic function $\nu$ such that $u=v$ a.e. in $\Omega$. However, as the following example shows $u$ need not be strictly plurisubharmonic.

EXAmPLe 1.9. Define $u: \mathbf{C}^{2} \rightarrow \mathbf{R}$ by $u\left(z_{1}, z_{2}\right)=\left|z_{1} z_{2}\right|^{2}$. For any nonzero $a \in \mathbf{C}^{2}$ and any nonnegative $\phi(\phi \not \equiv 0)$ in $\mathscr{D}\left(\mathbf{C}^{2}\right)$, we obtain from integrations by parts that

$$
\begin{aligned}
4(H(u ; \phi) a, \bar{a}) & =\sum_{j, k=1}^{2} \int_{\mathrm{C}^{2}}\left|z_{1} z_{2}\right|^{2} a_{j} \bar{a}_{k} \frac{\partial^{2} \phi(z)}{\partial z_{j} \partial \bar{z}_{k}} d z_{1} \wedge d z_{2} \wedge d \bar{z}_{1} \wedge d \bar{z}_{2} \\
& =\int_{\mathrm{C}^{2}}\left(\left|a_{1} z_{2}+a_{2} z_{1}\right|^{2}\right) \phi(z) d z_{1} \wedge d z_{2} \wedge d \bar{z}_{1} \wedge d \bar{z}_{2}>0,
\end{aligned}
$$

since $\left|a_{1} z_{2}+a_{2} z_{1}\right|^{2}>0$ except for a set of measure 0 in $\mathbf{C}^{2}$ and since $\phi>0$ on an open set in $\mathbf{C}^{2}$. By Theorem 1.4, $u$ is plurisubharmonic in $\mathbf{C}^{2}$ (this also follows from the fact that $u$ is the modulus of an entire function in $\mathbf{C}^{2}$ ); but $u$, vanishing on the variety $z_{1} \cdot z_{2}=0$, is not strictly plurisubharmonic.

From the case $n=1$ (Theorem 1.5), we obtain the following sufficient condition for strict plurisubharmonicity.

THEOREM 1.10. Let $u$ be locally integrable in an open set $\Omega \subset \mathbf{C}^{n}$. If for arbitrary $z \in \Omega$ and arbitrary nonzero $a \in \mathbf{C}^{n}$ the function $u_{z, a}(\tau)=u(z+a \tau)$ satisfies $\Delta u_{z, a}>0$ as a distribution in $\{\tau \in \mathbf{C}: z+a \tau \in \Omega\}$, then there exists a strictly plurisubharmonic function $\nu$ in $\Omega$ such that $u=\nu$ a.e. in $\Omega$.

PROof. Let $u_{\varepsilon}$ be the regularizations of $u$. Then from (1.8) we have that, for arbitrary nonnegative $\phi \in \mathcal{D}(\Omega)$ and any $z=z^{0}+a \tau \in \Omega$, $\left(\partial^{2} u_{\varepsilon}(z) / \partial \tau \partial \bar{\tau}\right) \phi(z) \geqslant 0$ if $\varepsilon$ is sufficiently small. From this we obtain by integration that $\left(H\left(u_{\varepsilon} ; \phi\right) a, \bar{a}\right) \geqslant 0$. Since $u_{\varepsilon} \rightarrow u$ in $\mathcal{Q}^{\prime}(\Omega)$, it follows that $(H(u ; \phi) a, \bar{a}) \geqslant 0$. Hence by Theorem 1.4 , there exists a plurisubharmonic function $\nu$ in $\Omega$ such that $u=\nu$ a.e. in $\Omega$. Now $\Delta \nu_{z, a}=\Delta u_{z, a}>0$, so by Theorem 1.6 we can conclude that $\nu$ is strictly plurisubharmonic in $\Omega$, which completes the proof.

We shall now give the analogues of Theorems 1.6, 1.7 and 1.10 for convex functions. For a detailed account of the analogies between convex functions and plurisubharmonic functions see [1] and [5].

Let $\mathscr{D}^{2} f$ denote the second distributional derivative of a locally integrable function $f$ on $(a, b)$. 
THEOREM 1.11. If $f$ is strictly convex ${ }^{4}$ on $(a, b)$, then $D^{2} f>0$ in $(a, b)$. Conversely, if $f$ is locally integrable on $(a, b)$ and $\mathscr{D}^{2} f>0$ in $(a, b)$, then there exists a strictly convex function $g$ in $(a, b)$ such that $f=g$ a.e. in $(a, b)$.

Proof. The proof, which follows from the analogue of Theorem 1.5 for convex functions (see [1], [4], or [5]), is essentially the same as the proof of Theorem 1.6. The details will not be repeated.

Let $\Omega$ be an open convex set in $\mathbf{R}^{n}$. For $\phi \in \mathscr{D}(\Omega)$, let $H_{\mathbf{R}}(f ; \phi)$ denote the distributional real Hessian of a locally integrable function $f$ in $\Omega$.

THEOREM 1.12. Let $\Omega$ be an open convex set in $\mathbf{R}^{n}$. If $f$ is strictly convex in $\Omega$, then

$$
\left(H_{\mathbf{R}}(f ; \phi) a, a\right)=\sum_{j, k=1}^{n} \int_{\mathbf{R}^{n}} f(x) a_{j} a_{k} \frac{\partial^{2} \phi(x)}{\partial x_{j} \partial x_{k}} d x>0
$$

for any nonzero $a \in \mathbf{R}^{n}$ and any nonnegative $\phi(\phi \not \equiv 0)$ in $\mathscr{D}(\Omega)$.

The proof of this theorem is similar to the proof of Theorem 1.17 and so is omitted.

EXAmple 1.13. Let $\Omega=\left\{\left(x_{1}, x_{2}\right) \in \mathbf{R}^{2}: x_{2}>0\right\}$ and define $f: \Omega \rightarrow \mathbf{R}$ by $f\left(x_{1}, x_{2}\right)^{\prime}=x_{1}^{4} / x_{2}$. Then

$$
\begin{aligned}
& \left(H_{\mathbf{R}}(f ; \phi) a, a\right) \\
& \quad=\int_{\Omega} 2 x_{1}^{2} / x_{2}\left[2 a_{1}^{2}+\left(2 a_{1}-a_{2} x_{1} / x_{2}\right)^{2}\right] \phi\left(x_{1}, x_{2}\right) d x_{1} d x_{2}>0
\end{aligned}
$$

for any nonzero $a \in \mathbf{R}^{2}$ and any nonnegative $\phi(\phi \neq 0)$ in $\mathscr{Q}(\Omega)$. By the analogue of Theorem 1.4 for convex functions (see [1] or [5]), $f$ is convex in $\Omega$. However, $f$ is not strictly convex since it vanishes for $x_{1}=0$.

THEOREM 1.14. Let $f$ be locally integrable in an open convex set $\Omega \subset \mathbf{R}^{n}$. If for arbitrary $x \in \Omega$ and arbitrary nonzero $a \in \mathbf{R}^{n}$ the function $f_{x, a}(t)=f(x+t a)$ satisfies $\mathscr{D}^{2} f_{x, a}>0$ as a distribution in $\{t \in \mathbf{R}: x+t a \in \Omega\}$, then there exists $a$ strictly convex function $g$ in $\Omega$ such that $f=g$ a.e. in $\Omega$.

The proof, which is similar to the proof of Theorem 1.10, is omitted.

REMARKS. (1) All of the corresponding theorems for strictly (plurisuperharmonic, superharmonic, and concave) functions are obvious and will not be stated.

(2) Note that $x^{4}+y^{4}$ defines a strictly convex function in $\mathbf{R}^{2}$ and a strictly subharmonic function in $\mathbf{C}$, and that $\left|z_{1}\right|^{4}+\left|z_{2}\right|^{4}$ defines a strictly plurisubharmonic function in $\mathbf{C}^{2}$. However, these functions are not strictly subharmonic (resp., strictly plurisubharmonic) in the sense of [2] or [6].

(3) In [7], neither of the functions $r(r(z, w)$ as defined on p. 266 and p. 268 of [7]) is strictly plurisubharmonic in any neighborhood of $(0,0)$, since in each case $r$ restricted to $z=0$ is harmonic. However, the function $u$ defined by

4 In the geometric sense. 


$$
u(z, w)=\operatorname{Re} w+|w|^{8}+\frac{15}{7}|w|^{2} \operatorname{Re} w^{6}+|z|^{8}+\frac{15}{7}|z|^{2} \operatorname{Re} z^{6}
$$

is strictly plurisubharmonic in $\mathbf{C}^{2}$, but the surface $S$ in $\mathbf{C}^{2}$ determined by $u=0$ also does not admit a holomorphic support function at $(0,0)$. This follows from Theorem $\mathrm{C}$ in [7].

\section{REFERENCES}

1. H. J. Bremermann, Complex convexity, Trans. Amer. Math. Soc. 82 (1956), 17-51. MR 18, 28.

2. L. Hörmander, An introduction to complex analysis in several variables, Van Nostrand, Princeton, N.J., 1966. MR 34 \#2933.

3. L Linear partial differential operators, 3rd ed., Die Grundlehren der math. Wissenschaften, Bd. 116, Springer-Verlag, New York, 1969. MR 40 \#1687.

4. L. Schwartz, Théorie des distributions, Publ. Inst. Math. Univ. Strasbourg, no. 9-10, Hermann, Paris, 1966. MR 35 \# 730.

5. V. S. Vladimirov, Methods of the theory of functions of complex variables, "Nauka", Moscow, 1964; English transl., M.I.T. Press, Cambridge, Mass., 1966. MR 30 \#2163; 34 \#1551.

6. P. Lelong, Plurisubharmonic functions and positive differential forms, Gordon and Breach, New York, 1969. MR 39 \#4436.

7. J. J. Kohn and L. Nirenberg, A pseudo-convex domain not admitting a holomorphic support function, Math. Ann. 201 (1973), 265-268. MR 48 \#8850.

Department of Mathematics, University of Missouri, Columbia, Missouri 65201 